\title{
Two-Way Relaying Cooperative Wireless Networks: Resource Allocation and Performance Analysis
}

\author{
Muhammad Abrar, Xiang Gui, and Amal Punchihewa
}

\begin{abstract}
Relay-based cooperative wireless networks have been widely considered one of the cost-effective solutions to meet the demands in future wireless networks. In order to maximize the overall sum-rate while maintaining proportional fairness among users, we investigate different resource allocation algorithms in two-way relay networks with analog network coding (ANC) protocol and time division broadcast (TDBC) protocol. The algorithms investigated are different from traditional proportional fairness schemes in terms of fairness and computational complexity as we have applied Access Proportional Fairness (APF) and Minimum Rate Proportional Fairness (MRPF) along with load balancing at the relays. A MATLAB simulation has been performed and simulation results show the effectiveness of these algorithms.
\end{abstract}

Keywords-relay network, relay protocol, resource allocation, two-way relaying, bidirectional relaying, Analog Network Coding, Time Division Broadcast

\section{INTRODUCTION}

C OOPERATIVE Relaying Wireless Communication has been proposed along with Orthogonal Frequency Division Multiplexing (OFDM) to meet the future demands in cellular communication networks. The idea of cooperative communication in wireless environment is more attractive due to the characteristic of diversity of wireless channels and the limited radio resources. Since the radio resources are limited in wireless systems, the efficient use of all these available resources is necessary to achieve better performance. Therefore, efficient and low complexity resource allocation algorithms are needed to meet these goals.

Due to the practical half-duplex nature of devices, there are two types of cooperative relaying proposed in the literature, namely one-way relaying (OWR) [1] and two-way relaying (TWR) [2]. Conventional cooperative networks are known as one-way relaying cooperative system. Due to half duplex operation of the relays in OWR network, two separate phases of transmission are required for the mobile terminal (MT) and the base station (BS) to exchange information via cooperation. Hence a total of four time slots are required to complete the exchange of information between the MT and the BS. In this type of relaying, there is a loss of half of the spectral efficiency as compared to full duplex relaying.

Full duplex relaying, in which a relay is able to transmit and receive on the same frequency at the same time, is practically more complex. Therefore, from a practical point of view,

M. Abrar and X. Gui are with the School of Engineering and Advanced Technology, Massey University, New Zealand (e-mail: M.Abrar@massey.ac.nz; X.Gui@massey.ac.nz).

A. Punchihewa is with Asia-Pacific Broadcasting Union, Kuala Lumpur, Malaysia (e-mail: amal@abu.org.my). half duplex relaying is preferred over full duplex operation even with this loss of spectral efficiency [3]. To overcome the spectral loss in OWR; two types of TWR have been proposed in the literature. The first type assumes that no direct link is available between MT and BS and only a relay link is available for transmission. Therefore, two time slots are required to complete the exchange of information between the MT and BS. The second type takes into account the direct link, and requires three time slots in order to complete the exchange of information [3]. These two types of Amplify and Forward (AF) based TWR are known as Analog Network Coding (ANC) protocol and Time Division Broadcast (TDBC) protocol, respectively [4].

The combination of cooperative relaying with multicarrier system provides promising design for next generation of wireless networks. As a multicarrier transmission technique, OFDM has already been employed in a number of communication standards including Digital Audio Broadcasting (DAB), Digital Video Broadcasting (DVB), Wireless LAN standards and IEEE 802.16 Broadband Wireless Access System. OFDM is also considered to be employed in next generation relaying wireless standards such as IEEE $802.16 \mathrm{j}$, IEEE.802.16m and 3GPP LTE-A [5].

In recent years there has been an extensive amount of research undertaken and published in the area of cooperative networks. The initial work on cooperative networks was focused on OWR only; but now TWR has been attracting a lot of interest from researchers due to its high spectral efficiency.

In a general resource allocation problem as addressed in the literature, the main focus resides in resource allocation without any traffic or load balancing consideration. In [4], authors have addressed the relay selection problem for TWR networks. The closed form expression for outage probability is derived. A single-pair network is considered, therefore there is no multi-user interference. A similar network configuration is used in [6] to address the relay selection problem in bidirectional relaying with unknown channel state information (CSI). The problem of resource allocation in TWR has been addressed in [7] for a two-terminal network with multiple relays. A fairness constraint is imposed on relays with maximum sum capacity as an objective function. In [8], a hierarchical protocol for OWR and TWR is proposed. The transmission mode of each MT as well as relay is already assigned, either direct or relaying. The joint resource allocation problem is formulated under total power constraints. Power allocation with data rate fairness is studied in [9] for Amplify-andForward (AF) and Decode-and-Forward (DF) protocols. 
In [10], a joint optimization problem of sub-carrier assignment and relay selection in multiple user pairs for bidirectional relaying communication is addressed. A graph theoretical approach is applied to solve this problem. In [11], resource allocation problem with sub-carrier pairing in OFDM-based TWR relay network is formulated as a mixed integer programming problem. By using dual method, the optimization problem is solved. In [12], we investigate the subcarrier allocation problem in AF protocol based OWR networks.

In conventional non-cooperative cellular networks, several load balancing aware algorithms have been reported [13]-[15]. However, these algorithms cannot be used directly for relaybased networks due to dual-hop or multi-hop transmission in relay networks [16]. In [16], authors provide fairnessaware joint routing and scheduling technique for downlink OWR networks. While in [17], an optimal mobile association and load balancing has been introduced in downlink OWR cooperative networks. Some other individual work on load balancing can be found in [18]-[20].

\section{A. Motivation and Contribution}

A relay-based cooperative wireless network has been widely considered one of the cost-effective solutions to meet the demands in future wireless networks. Most of the currently available resource allocation methods for relay networks are focused on maximizing system capacity either with proportional fairness or minimization of power. Some individual work on load balancing in one-way relay network has also been reported in the literature. In this paper, a fairness-aware joint load balancing and proportional fairness based resource allocation is investigated in TWR cooperative networks. The objective function is to maximize overall sum-rate under the load balancing and fairness constraints. We investigate load balancing with access proportional fairness (LB-APF) and load balancing with minimum rate proportional fairness (LBMRPF) in TWR with both ANC and TDBC protocols. To the best of the authors' knowledge, such a joint load balancing and proportional fairness based resource allocation in TWR with both protocols has not been investigated so far in the literature.

\section{B. Organization}

The rest of the paper is organized into four further sections. Section II presents the system model. A brief description of ANC and TDBC protocols is also presented in this section. In Section III, resource allocation schemes and problem formulation are presented. Furthermore, numerical results with simulation are illustrated in Section IV. Finally, conclusion is provided in Section V.

\section{System Model AND PROtocol Description}

\section{A. System Model}

A TWR transmission is considered in relay-based mobile cooperative network. The system model consists of a single $\mathrm{BS}, R$ number of relay terminals (RTs) and $M$ number of mobile terminals (MTs). Each terminal is equipped with a single

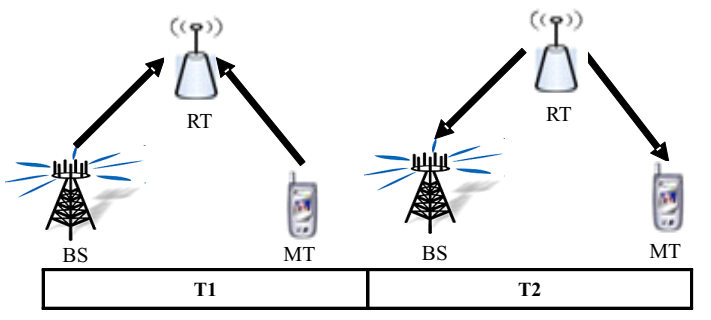

Fig. 1. TWR-ANC assisted transmission model.

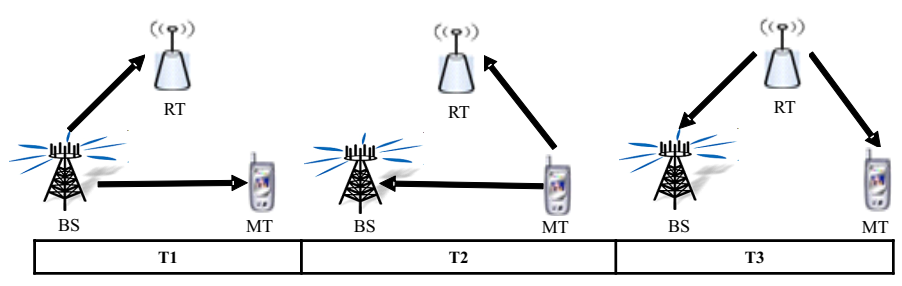

Fig. 2. TWR-TDBC assisted transmission model.

antenna. Each MT makes a pair with BS for communication. Time Division Duplex (TDD) is used to achieve separation between uplink and downlink transmission to and from relay respectively as all relays are working in half duplex mode and adopt AF protocol. In Long Term Evolution (LTE) system, a chunk consisting of twelve consecutive OFDM sub-carriers, known as the Resource Block (RB), is introduced. Therefore an $\mathrm{RB}$ is the minimal unit that is allowed to be allocated to one user [21]. We assume that there are $K$ RBs available in the cell and in our analysis we use RBs instead of sub-carriers.

\section{B. Protocol Description}

\section{1) ANC Protocol}

In ANC protocol as shown in Fig. 1, both MT and BS transmit their signal to the relay during the first time slot. A relay receives the combined signal of MT and BS due to broadcast nature of wireless channel. The relay amplifies this combined signal and then retransmits to both MT and BS in the second time slot.

\section{2) TDBC Protocol}

In TDBC protocol as shown in Fig. 2, BS transmits its signal to both MT and relay during the first time slot, while MT transmits its signal to both BS and relay in the second time slot. In the third time slot the relay amplifies the combined signal of BS and MT and retransmits to both MT and BS.

In both protocols, the received signal at MT and BS consists of their own transmitted signal known as the self-interference. With the knowledge of the channel and its own signal, this selfinterference can be subtracted from the received signal [2].

\section{Mathematical Description}

The achievable rates from BS to MT and from MT to BS in ANC protocol of the $m^{t h} \mathrm{MT}$ via the $r^{t h}$ relay on the $k^{t h}$ $\mathrm{RB}$ is given by (1) and (2), respectively [22]

$$
R_{m, r, k}^{A N C}=\frac{1}{2} \log _{2}\left(1+\gamma_{m, r, k}^{A N C}\right)
$$




$$
R_{b, r, k}^{A N C}=\frac{1}{2} \log _{2}\left(1+\gamma_{b, r, k}^{A N C}\right)
$$

The factor $\frac{1}{2}$ appears here due to the half-duplex operation of relays. It means relays transmit and receive in two different time slots while $\gamma_{m, r, k}^{A N C}$ and $\gamma_{b, r, k}^{A N C}$ are the received SNRs at the MT and the BS, respectively. The instantaneous sum-rate is then given by

$$
\begin{gathered}
R_{r, k}^{A N C}=R_{m, r, k}^{A N C}+R_{b, r, k}^{A N C} \\
=\frac{1}{2} \log _{2}\left(1+\gamma_{m, r, k}^{A N C}\right)+\frac{1}{2} \log _{2}\left(1+\gamma_{b, r, k}^{A N C}\right)
\end{gathered}
$$

Let $\gamma_{m, r, k}^{T D B C}$ and $\gamma_{b, r, k}^{T D B C}$ represent the SNRs at MT and BS respectively for TDBC protocol, therefore the instantaneous sum-rate of TWR-TDBC protocol can be written as

$$
R_{r, k}^{T D B C}=\frac{1}{3} \log _{2}\left(1+\gamma_{m, r, k}^{T D B C}\right)+\frac{1}{3} \log _{2}\left(1+\gamma_{b, r, k}^{T D B C}\right)
$$

The factor $\frac{1}{3}$ shows that transfer of information between two nodes is completed in three time slots.

The achievable sum-rate of the network over all MTs and $\mathrm{RBs}$ using any protocol is given by

$$
r=\sum_{m=1}^{M} \sum_{r=1}^{R} \sum_{k=1}^{K} \delta_{m, r, k} R_{m, r, k}
$$

where $\delta_{m, r, k}$ is used as the binary integer RB assignment indicator variable. If the $k^{\text {th }} \mathrm{RB}$ is assigned to the $m^{\text {th }} \mathrm{MT}$ on the $r^{t h} \mathrm{RT}$, then $\delta_{m, r, k}$ is equal to one, otherwise it is equal to zero. The term $R_{m, r, k}$ is equal to $R_{m, r, k}^{A N C}$ or $R_{m, r, k}^{T D B C}$ depending on the system protocol.

\section{Problem Formulation And Description}

In order to maximize the system throughput while maintaining load balancing at relays and proportional fairness among users, a binary integer linear programming (BILP) optimization problem is formulated as

$$
\text { Maximize } \quad \sum_{m=1}^{M} \sum_{r=1}^{R} \sum_{k=1}^{K} \delta_{m, r, k} R_{m, r, k}
$$

Subject to the following constraints:

C1: RB-Allocation Constraint: The RB-Allocation constraint is that each RB can be used only by one RT to avoid intra-cell interference.

$$
\begin{gathered}
\sum_{m=1}^{M} \sum_{r=1}^{R} \delta_{m, r, k} \leq 1, \forall k, \\
\delta_{m, r, k} \in\{0,1\}, \forall(m, r, k)
\end{gathered}
$$

C2: Load-Balancing Constraint: The load-balancing constraint is that RBs are equally distributed among all relays.

$$
\sum_{m=1}^{M} \sum_{k=1}^{K} \delta_{m, r, k} \geq \mu, \forall r
$$

where $\mu=[K / R]$ is the minimum number of $\mathrm{RBs}$ to be allocated to any RT, where $[\mathrm{x}]$ denotes the integer part of x. If $K \bmod R=0$, each $\mathrm{RT}$ will be assigned exactly $K / R$ RBs.

C3: MRPF Constraint: This constraint ensures that each MT meets the minimum date rate requirement. Let $r_{m \text {,min }}$ be the minimum rate requirement (MRR) for the $m^{\text {th }}$ MT, then

$$
\sum_{r=1}^{R} \sum_{k=1}^{K} \delta_{m, r, k} R_{m, r, k} \geq r_{m, \min }, \forall m
$$

C4: APF Constraint: This constraint is applied when we need to distribute all RBs equally among all MTs.

$$
\sum_{r=1}^{R} \sum_{k=1}^{K} \lambda_{m, r, k} \geq \lambda, \forall m
$$

where $\lambda=[K / M]$ is the minimum number of RBs to be allocated to any MT. If $K \bmod M=0$, each MT will be assigned exactly $K / M$ RBs.

This BILP optimization problem is non-polynomial in time and the computational complexity of such problem can be expressed as $O(R \times M)^{K}$. With increasing number of $R, M$ and $K$, the computational complexity might reach prohibitive limits in practical systems. Therefore, a low complexity algorithm is necessary to solve this type of optimization problem.

\section{A. Hungarian Algorithm Based Resource Allocation}

In this sub-section, we solve our BILP optimization problem by using Hungarian Algorithm (HA) [23]. The HA is a oneto-one optimization solver for assignment problems. The main reason of using this algorithm is that load balancing at relays is achieved inherently with this algorithm.

The following sub-steps are involved in applying HA to our BILP problem.

1) The demand metric showing the sum-rate on each $R B$ of each RT is calculated as the maximum of $M$ links as

$$
D_{k, r}=\max _{\forall m}\left\{\omega_{m, r, k} R_{m, r, k}\right\},
$$

where $\omega_{m, r, k}$ is the binary integer variable as proportional fairness index (PFI) for the $m^{\text {th }}$ MT on the $k^{\text {th }}$ $\mathrm{RB}$ using the $r^{\text {th }}$ relay.

2) The total of $R$ demand metrics on each RB has been calculated. By applying HA to the $K \times R$ demand matrix as shown in Fig. 3, the algorithm solves a one- toone optimization problem. In each iteration, $R$ RBS are

\begin{tabular}{|c|c|c|c|c|}
\hline & $\mathbf{R T}_{1}$ & $\mathbf{R T}_{2}$ & $\cdots$ & $\mathbf{R T}_{\mathrm{R}}$ \\
\hline $\mathbf{K}_{1}$ & $\mathbf{D}_{1,1}$ & $\mathbf{D}_{1,2}$ & $\cdots$ & $\mathbf{D}_{1, \mathrm{R}}$ \\
\hline $\mathbf{K}_{2}$ & $\mathbf{D}_{2,1}$ & $\mathbf{D}_{2,2}$ & $\cdots$ & $\mathbf{D}_{2, \mathrm{R}}$ \\
\hline$\ldots$ & $\ldots$ & $\ldots$ & $\cdots$ & $\cdots$ \\
\hline$\cdots$ & $\cdots$ & $\cdots$ & $\cdots$ & $\cdots$ \\
\hline $\mathbf{K}_{\mathrm{K}}$ & $\mathbf{D}_{\mathrm{K}, 1}$ & $\mathbf{D}_{\mathrm{K}, 2}$ & $\cdots$ & $\mathbf{D}_{\mathrm{K}, \mathrm{R}}$ \\
\hline
\end{tabular}

Fig. 3. Snapshot of one iteration of HA Matrix. 

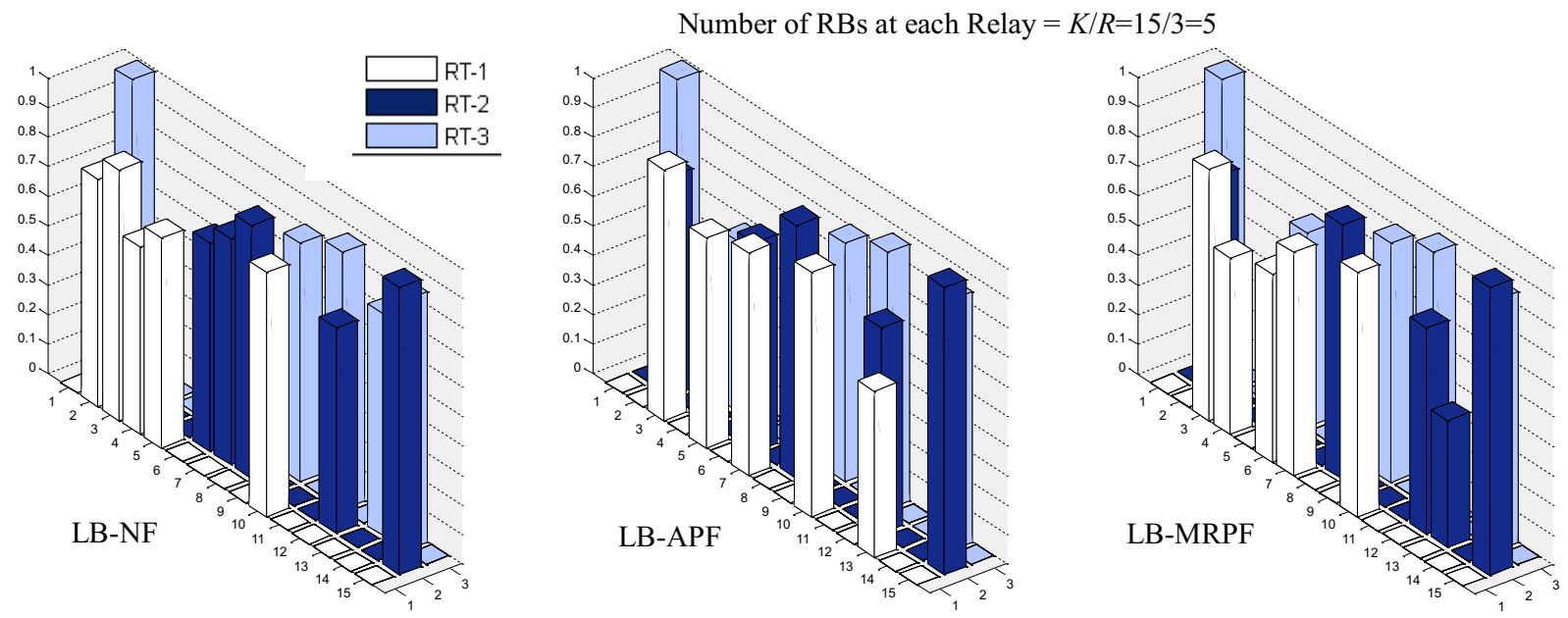

Fig. 4. Load balancing at Relays in TWR-ANC Protocol (x-axis: IDs of RTs, y-axis: IDs of RBs, z-axis: Achieved sum-rate).
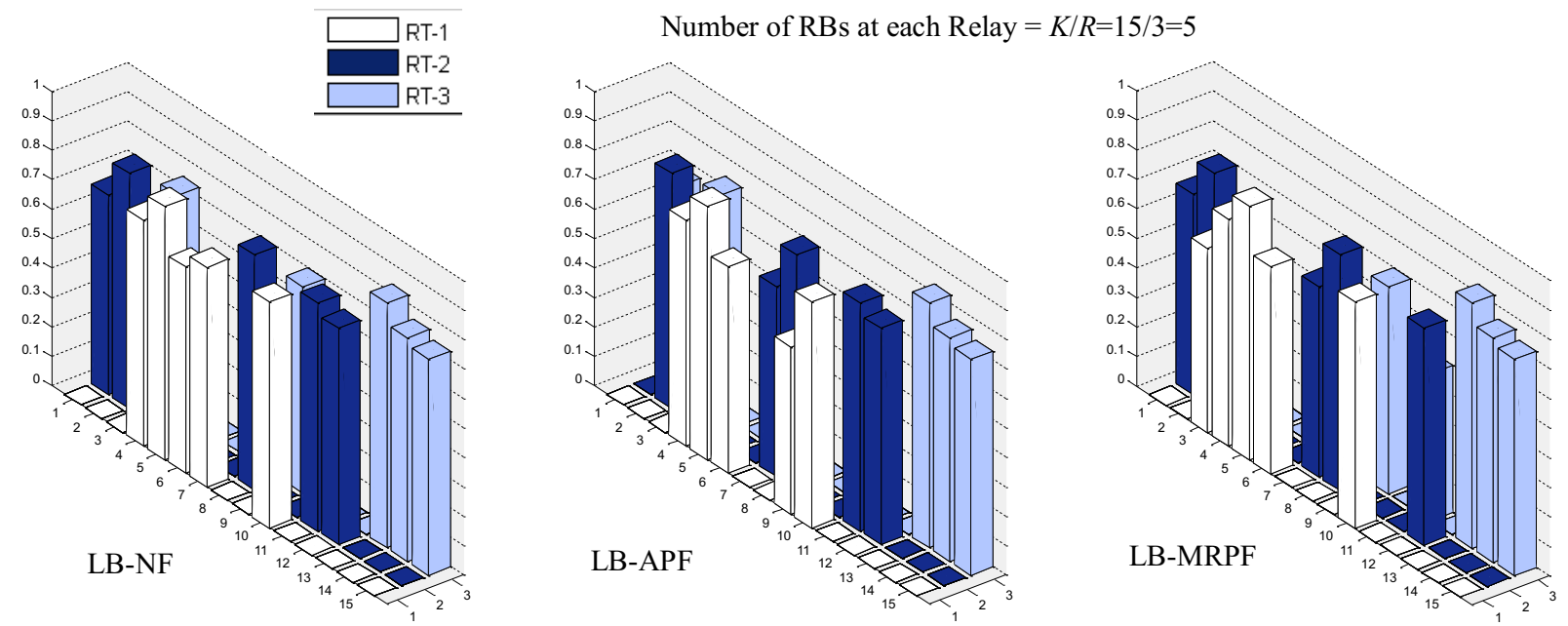

Fig. 5. Load balancing at Relays in TWR-TDBC Protocol (x-axis: IDs of RTs, y-axis: IDs of RBs, z-axis: Achieved sum-rate).

allocated. Therefore total $K / R$ iterations are needed to allocate all the $K$ RBs. To understand how HA works, please refer to [21].

3) The rows with assigned RBs are eliminated and MTs are marked with assigned RBs.

4) The constraint (6-c) or (6-d) is checked for LB-MRPF and LB-APF, respectively. The PFI of MTs satisfying these constraints are set equal to zero.

5) The steps 1-4 are repeated until all RBs are assigned or all MTs have achieved MRR.

6) If RBs are still available in LB-MRPF, the process is repeated except step 4 until all RBs are assigned.

\section{B. Computational Complexity}

By using HA, the computational complexity has been significantly reduced. The polynomial complexity of one iteration of HA is $O\left(K_{n}^{3}\right)$ [15], [21], where $K_{n}$ is the number of unassigned RBs. With $K / R$ iterations, the complexity of the whole algorithm is $O\left(K^{4} / R\right)$. The pseudocode for the HA based resource allocation algorithm is given in Algorithm-1.

\section{NumERiCAL RESUltS AND AsSUMPTIONS}

In this section, we present and compare numerical results with the help of computer simulations to evaluate the performance of resource allocation algorithms described in sectionIII. In this simulation we assume that there is no direct link available between BS and MTs for transmission and we also assume random distribution of MTs. All the channels are simulated as Rayleigh flat fading. We consider that all channels remain constant for one complete transmission. For simplicity, it is assumed that CSI is known to all nodes. All noise variances are identical and reciprocal channels are assumed. The proposed algorithms have been evaluated in terms of total achievable system sum-rate and achievable individual sum-rate of each pair.

Figures 4 and 5 show the RBs assigned to each relay in three different algorithms for ANC and TDBC protocols, respectively. It can be observed that each relay can occupy 5 RBs which is equal to $K / R$, with $K=15$ and $R=3$. 


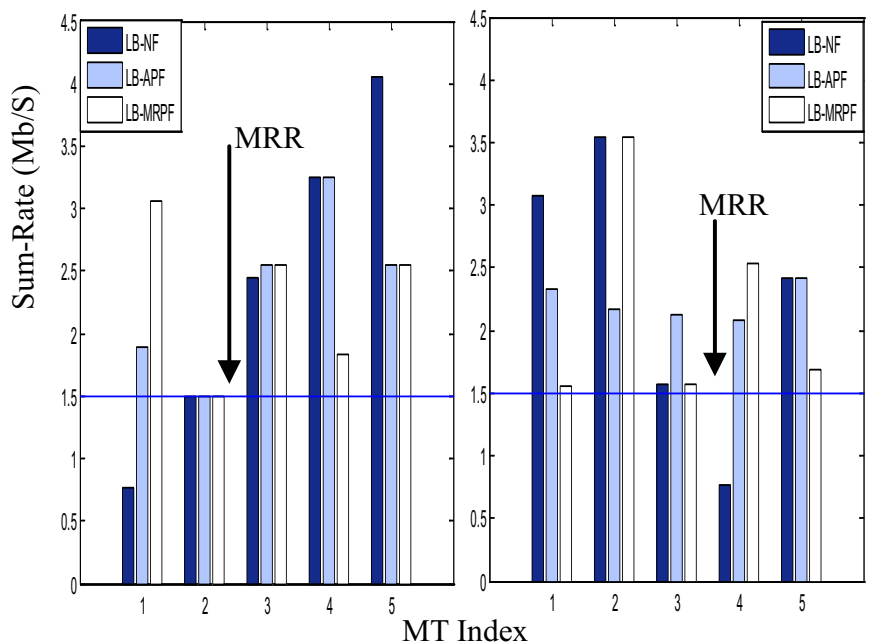

(a) ANC

(b) TDBC

Fig. 6. Individual sum-rate for each MT-BS pair in TWR.

\begin{tabular}{l} 
Algorithm-1 \\
\hline 1: Set MTs $=\{1,2,3, \ldots, M\}, \mathrm{RTs}=\{1,2,3, \ldots, R\}$, \\
RBs $=\{1,2,3, \ldots, K\}$ \\
$R^{\prime}=$ Size of RTs, $K^{\prime}=$ Size of RBs \\
2: for $k=1: K^{\prime}$ \\
3: for $r=1: R^{\prime}$ \\
4: $D_{k, r}=\max _{m}\left\{\omega_{m, r, k} R_{m, r, k}\right\} m \in\{1,2, \ldots, M\}$ \\
5: end for \\
6: end for \\
7: D $=\left\{D_{k, r}\right\}, r=\left\{1,2, \ldots, R^{\prime}\right\}, k=\left\{1,2, \ldots, K^{\prime}\right\}$, \\
as shown in Fig. 3 \\
8: for rounds $=1: K^{\prime} / R^{\prime}$ \\
9: Hungarian $(\mathrm{D}) \Rightarrow$ ARBs $=$ Set of assigned RBs \\
10: Do RBs $=$ RBs - ARBs \\
11: $K^{\prime}=$ Size of RBs \\
12: Do for LB - APF \\
13: AMTs1 $=$ Set of MTs Satisfying \\
$\sum_{r=1}^{R} \sum_{k=1}^{K} \lambda_{m, r, k} \geq \lambda, \forall m \in$ MTs \\
14: Do MTs $=$ MTs - AMTs 1 \\
15: Do for LB - MRPF \\
16: AMTs2 $=$ Set of MTs satisfying \\
$\sum_{r=1}^{R} \sum_{k=1}^{K} \delta_{m, r, k} R_{m, r, k} \geq r_{m, \text { min }}, \forall m \in$ MTs \\
17: Do MTs $=$ MTs-AMTs2 \\
18: MTs $=\emptyset$ and $K^{\prime} \neq 0$ go to 22 \\
19: if $K^{\prime}=0$ go to 23 \\
20: end if; end if \\
21: end for \\
22: Repeat only $3-11$ until $K^{\prime}=0$ \\
23: Exit \\
\hline
\end{tabular}

By distributing the traffic load among relays, we reduce the processing delays at relays. Figure 6 shows the individual achievable sum-rate for each pair in ANC and TDBC protocols. In this case of individual sum-rate, LB-MRPF outperforms load balancing with no fairness (LB-NF). The MRR for all MTs is achieved in LB-MRPF and LB-APF,

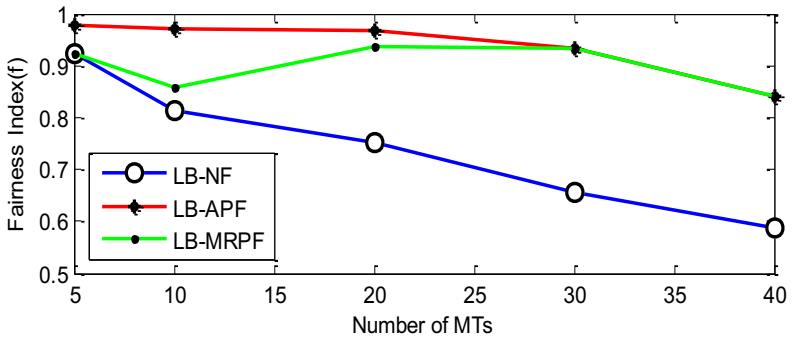

(a) TWR-ANC

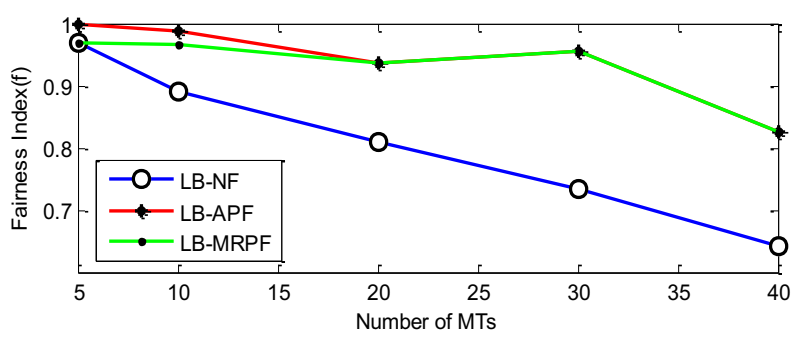

(b) TWR-TDBC

Fig. 7. Jain's fairness index with different number of MTs.

while the LB-NF is not able to meet MRR for all MTs in both protocols. In Fig. 6a it can be observed that the sum-rate of MT-1 with LB-NF algorithm is less than required MRR while it is also clear in Fig. 6b that MT-4 cannot achieve MRR with LB-NF algorithm. Even though the MRR is also achieved in LB-APF, but overall sum rate of LB-APF is lower than that of LB-MRPF scheme which is shown in Fig. 8 and Fig. 10.

Jain's fairness index has been widely used to determine the proportional fairness among users [15]. It is given by

$$
f=\frac{\left[\sum_{i=1}^{K} r_{i}\right]^{2}}{K \sum_{i=1}^{K} r_{i}^{2}}
$$

where $r_{i}$ is the normalized sum-rate for the $i^{\text {th }}$ user. The value of this index ranges from 0 (worst case) to 1 (best case).

Figures $7 \mathrm{a}$ and $7 \mathrm{~b}$ show that fairness index remains around 90\% in both LB-MRPF and LB-APF while it drops significantly in LB-NF with increasing number of users in both ANC and TDBC protocols, respectively.

The system sum-rate performance against different number of RBs and MTs for both protocols can be observed in Fig. 8 and Fig. 10, respectively while Fig. 9 shows the magnified image of the results shown in Fig. 8. It can easily be observed that the overall sum-rate of LB-NF is always the highest for different number of RBs or MTs. This is due to the fact that this algorithm assigns RBs to MTs with the best channel gains, hence increasing overall sum-rate at the expense of proportional fairness among MTs. In contrast, LB-APF gives lower sum-rate than others because it ensures access proportional fairness among MTs by enforcing assignment of equal number of RBs, which may compromise on overall and individual sum-rates. A compromise between sum-rate and fairness has been made in LB-MRPF and a good tradeoff 


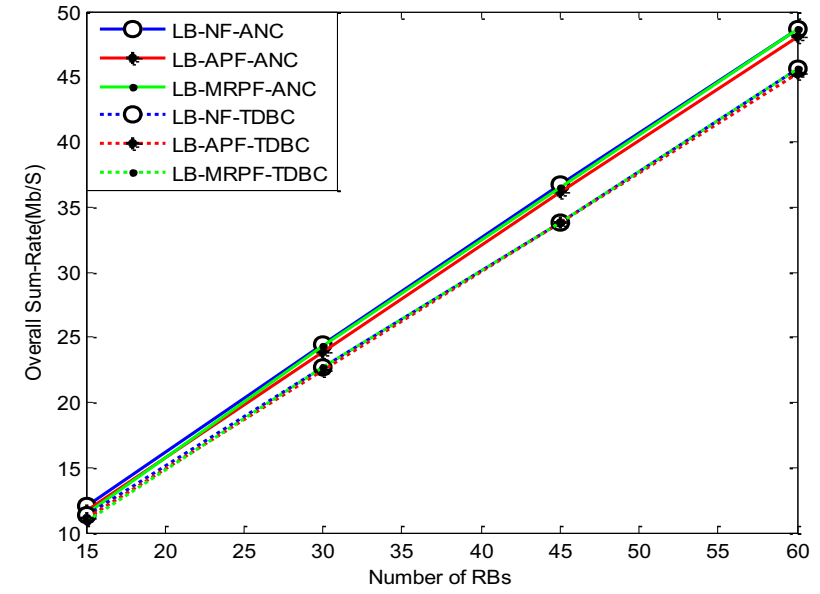

Fig. 8. Overall sum-rate versus the number of RBs.

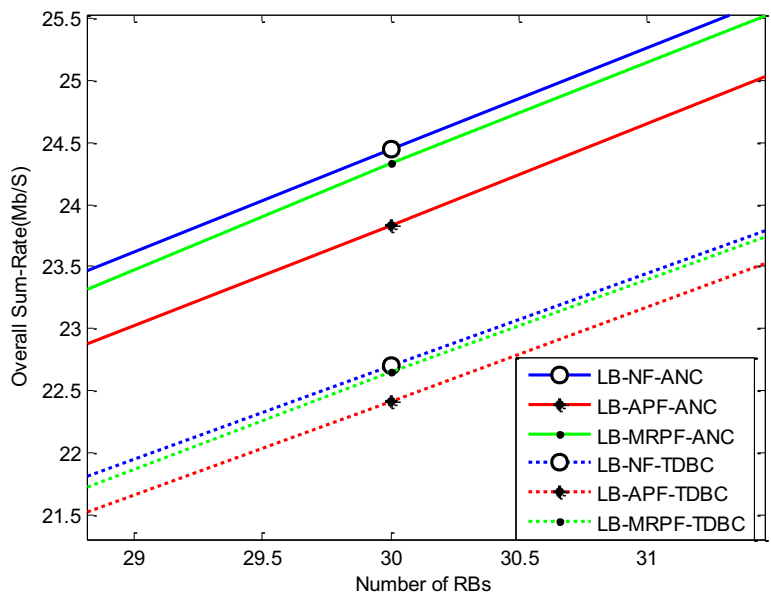

Fig. 9. Overall sum-rate versus the number of RBs (magnified).

between overall sum-rate and individual sum-rate can be observed.

In Fig. 10 we can observe that cell throughput increases with an increase in the number of MTs. This indicates that RBs are assigned to MTs with the best channel gains in all schemes with or without proportional constraints.

There is also some throughput loss and inconsistency versus the increase in the number of MTs. This is due to the fact that we have fixed the number of RBs at 60 for all numbers of MTs. For good ratio of number of MTs over RBs, there is a remarkable increase in cell throughput, like when there is a number of MTs $=10$. On the other hand if number of MTs increasing more, the gain in throughput is not sufficiently increasing because the ratio of MTs over RBs decreasing and RBs are not available for some MTs. This also reflects in Fig. 7 where fairness index drops significantly for large number of MTs when we have fix number of RBs.

\section{CONCLUSIONS}

Efficient resource allocation algorithms are required to meet the demands of future relay-based wireless networks. A fairness-aware joint load balancing and proportional fairness based resource allocation has been investigated in two-way relaying cooperative networks with both ANC and TDBC

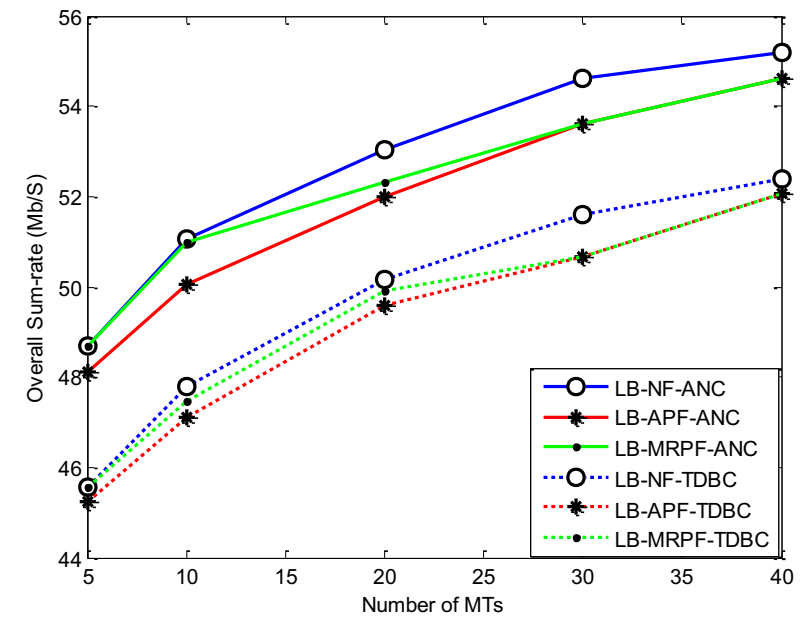

Fig. 10. Overall sum-rate versus the number of MTs.

protocols in this paper. A BILP optimization problem is formulated to maximize the overall system sum-rate. To reduce computational complexity, Hungarian Algorithm based resource allocation has been proposed which inherently provides load balancing at relays. Finally, we remark that, while this paper considers sum-rate at equal power allocation, the consideration of individual rates of BS and MT with optimized power remains an interesting issue to be explored.

\section{REFERENCES}

[1] J. N. Laneman, et al., "Cooperative diversity in wireless networks: Efficient protocols and outage behavior," IEEE Transactions on Information Theory, vol. 50, pp. 3062-3080, 2004.

[2] R. Boris and W. Armin, "Spectral efficient protocols for half-duplex fading relay channels," IEEE Journal on Selected Areas in Communications, vol. 25, pp. 379-389, 2007.

[3] A. Agustin, et al., "Protocols and Resource Allocation for the TwoWay Relay Channel with Half-Duplex Terminals," in IEEE International Conference on Communications, 2009. ICC '09., 2009, pp. 1-5.

[4] J. MinChul and K. Il-Min, "Relay Selection with ANC and TDBC Protocols in Bidirectional Relay Networks," IEEE Transactions on Communications, vol. 58, pp. 3500-3511, 2010.

[5] R. Prasad, OFDM for wireless communications systems. Bostan, London: Artech House, 2004.

[6] S. Lingyang, et al., "Joint Relay Selection and Analog Network Coding Using Differential Modulation in Two-Way Relay Channels," IEEE Transactions on Vehicular Technology, vol. 59, pp. 2932-2939, 2010.

7] K. Yookeun, et al., "Resource Allocation for Two-Way OFDM Relay Networks with Fairness Constraints," in Vehicular Technology Conference Fall (VTC 2009-Fall), 2009, pp. 1-5.

[8] K. Jitvanichphaibool, et al., "Optimal Resource Allocation for Two-Way Relay-Assisted OFDMA," EEE Transactions on Vehicular Technology, vol. 58, pp. 3311-3321, 2009.

[9] M. Pischella and D. L. Ruyet, "Optimal Power Allocation for the TwoWay Relay Channel with Data Rate Fairness," IEEE Communications Letters, vol. 15, pp. 959-961, 2011.

[10] Y. Liu and M. Tao, "Optimal Channel and Relay Assignment in OFDMBased Multi-Relay Multi-Pair Two-Way Communication Networks," IEEE Transactions on Communications, vol. PP, pp. 1-5, 2011.

[11] Z. Hao, et al., "Resource Allocation with Subcarrier Pairing in OFDMA Two-Way Relay Networks," IEEE Wireless Communications Letters, vol. 1, pp. 61-64, 2012.

[12] M. Abrar, et al., "Sub-carrier allocation for downlink multi-user OFDM cooperative cellular networks," in 6th International Conference on Broadband and Biomedical Communications (IB2Com), 2011, pp. 6367.

[13] M. Jie, et al., "Joint Power and Bandwidth Allocation Algorithm with QoS Support in Heterogeneous Wireless Networks," IEEE Communications Letters, vol. 16, pp. 479-481, 2012. 
[14] C. Yao-Hsing and C. Chung-Ju, "A Balanced Resource Scheduling Scheme With Adaptive Priority Thresholds for OFDMA Downlink Systems," IEEE Transactions on Vehicular Technology, vol. 61, pp. 1276-1286, 2012.

[15] X. Lexi, et al., "Cooperative load balancing for OFDMA cellular networks," 18th European Wireless Conference, European Wireless 2012. EW., pp. 1-7, 2012.

[16] M. Salem, et al., "Fairness-aware radio resource management in downlink OFDMA cellular relay networks," IEEE Transactions on Wireless Communications, vol. 9, pp. 1628-1639, 2010.

[17] Y. Yi, et al., "Mobile association and load balancing in a cooperative relay cellular network," IEEE Communications Magazine, vol. 49, pp. 83-89, 2011.

[18] P. Kolios, et al., "Load Balancing via Store-Carry and Forward Relaying in Cellular Networks," in EEE Global Telecommunications Conference (GLOBECOM 2010), 2010, pp. 1-6.
[19] S. D. Jun and J. J. Wook, "A Path Selection Scheme Considering Traffic Load for IEEE 802.16j Mobile Multi-Hop Relay Networks," in 6th International Conference on Wireless Communications Networking and Mobile Computing (WiCOM), 2010, pp. 1-5.

[20] N. Ghaboosi and A. Jamalipour, "A Cooperative Cellular Architecture with Emphasis on Traffic Load Balancing," in IEEE Wireless Communications and Networking Conference (WCNC), 2010, pp. 1-6.

[21] 3rd Generation Partnership Project, Technical Specification Group RadioAccess Network; Physical Layer Aspect; 3GPP TS 36.201 version 10.0.0.

[22] P. Jingjing and T. See, "Rate performance of AF two-way relaying in low SNR region," IEEE Communications Letters, vol. 13, pp. 233-235, 2009.

[23] H. W. Kuhn, "The Hungarian method for the assignment problem," Naval Research Logistic Quarterly, vol. 2, pp. 83-97, 1955. 